broad agreement in the geophysical community has yet to be proposed. The gravitational effect of sinking slabs, pressure effects from mid-ocean ridges, deeper convective patterns as manifested either by broad scale motions or by narrow plumes are all under study, but the nut is a tough one. Sykes and Sbar look at these processes and compare their results with the implications of separate processes. In no case is there anything like good agreement, so they refrain from coming down in favour of any one explanation. Instead, they have provided geophysicists with a new and powerful constraint on dynamical models of plate tectonics, and in the long run this is probably more important.

D. D.

\section{Antigens on Trophoblasts}

MODERN immunology has more than its share of riddles and paradoxes, and one of the most intriguing of these is the failure of the mother immunologically to reject her foetus, in spite of the fact that they have-in outbred animals and humans - a relationship that is biologically akin to that of an allograft and its host. The solution to this puzzle is important for reasons which go beyond satisfying simple immunological curiosity: for one thing, an understanding of the methods nature uses to prevent foetal-allograft rejection could go a long way in helping to solve the rejection problem which plagues the transplant surgeon.

Inevitably, an array of explanations has been put forward; these include depressed maternal immune responsiveness, immunological 'enhancement' mediated by serum blocking factors, placental barrier function and weak foetal immunogenicity. None of these theories, however, commands wide general acceptance.

The presence of antigens on trophoblast cells is a case in point. It is known that the mother can, and often does, make antibodies to some of the alloantigens of her foetus even though maternal and foetal circulations are entirely separate. To resolve this problem much attention has been paid to the trophoblast layer of the placenta as it is the only location where there is mutual surface contact between maternal and foetal tissues. Hence the special interest in the subject of antigens such as histocompatibility or blood group antigens on trophoblast cells. In short, is the trophoblast immunologically neutral or can it sensitise the mother to histocompatibility and blood group antigens? Most studies have been negative in detecting histocompatibility antigens on trophoblast cells, but enough have been successful to keep the issue continually embroiled in controversy. One popular notion is that antigens on trophoblast cells are 'masked' by a thick sialo-mucinous glyocalyx. The experiments in which this cell coat has been enzymatically removed with neuraminidase have not, however, yielded consistent evidence of the exposure of antigens.

On page 329 of this issue of Nature, Loke and Ballard address themselves to the problem of blood group antigens (type A) on human trophoblast cells. The authors use three different techniques to try and detect such antigens: first, a mixed agglutination method using trophoblast cells coated with anti-A serum and group A red blood cells; second, an antiglobulin method using group A red cells and trophoblast both being coated with anti-A with a rabbit anti-human globulin acting as a cross-linking bridge molecule; and, third, an electron microscopic method using a coating of ferritin-labelled anti-human globulin antiserum on cells previously incubated with anti-A antiserum. The second method yielded an unequivocally positive result and the third, though permitting some visualisation of ' $A$ ' antigens, suggested they were present in small amount or density. Loke and Ballard speculate that, because the surface density of antigens on trophoblast cells is low and their spacial topography such, only very sensitive methods will be successful in their detection. Low antigen density (or antigen inaccessibility) may result in insufficient antibody being taken up to form stable bridges directly with the indicator cells in the mixed agglutination method. Addition of another link introduced in the form of an antiglobulin, however, results in visible agglutination between trophoblast cells and indicator red cells.

The question now is what, if anything, is the biological importance of low antigen density on trophoblast cells? Loke and Ballard suggest that since antigenic density of target cells may be an important factor in the phenomenon of immunological enhancement, the fixation of antibody on surface antigens which are sparsely distributed may lead to a 'blocking' or enhancing effect, thus helping to ensure survival of the foetus. As with so many biological problems for which there are various and conflicting solutions it seems that none of them will prove uniquely to be correct.

From a Correspondent

\section{Cosmological Coincidences}

Two cosmological coincidences are discussed by Cavallo on page 313 of this issue of Nature. One of them is the Dirac relationship between fundamental constants.

It is well known that in situations where well-founded theoretical arguments are difficult, a dimensional argument (if it leads to a unique answer at all) will give an answer which is not usually out by a large factor. The reason is that nature's dimensionless numbers do not differ by many orders of magnitude from unity. Even the sign structure constant (about 1/137) is something of an exception.

Two very striking exceptions are numbers of order $10^{40}$. One is the ratio of electromagnetic repulsion to gravitational attraction between two electrons. The other is a dimensionless combination involving Hubble's constant (which determines the rate of the Universe's expansion and is thought to be related to its age so that the combination could be thought of as a way of expressing the age of the Universe in "natural" units).

The second of these numbers is not well enough determined to refute the hypothesis that it is identically equal to the first. This is a striking coincidence. It was proposed by Dirac in 1937 that strict equality might hold between the two numbers and that gravity weakens to maintain the equality as the Universe gets older.

The second coincidence was expressed dramatically in 1969 in a paper by Hawking (Cavallo's reference 3) called "Is the Universe Rotating?". It is not a meaningless question in spite of the rejoinder "Relative to what?" because rotation produces local mechanical effects. It is a coincidence that the frame of reference in which there are no such mechanical effects is the same as that in which the distant galaxies are not rotating around us. 